\title{
Lessons To Be Learned: Identifying High-Risk Medication and Circumstances In Patients At Risk For Suicidal Self-Poisoning
}

\section{Stefanie Geith ( $\square$ Stefanie.Geith@tum.de)}

Technical University of Munich Hospital Rechts der Isar Medical Clinic and Polyclinic II: Klinikum rechts der Isar der Technischen Universitat Munchen Klinik und Poliklinik fur Innere Medizin II Gastroenterologie https://orcid.org/0000-0002-2726-2823

\section{Christiane Didden}

LMU: Ludwig-Maximilians-Universitat Munchen, Department of Sociology

\section{Christian Rabe}

Division of Clinical Toxicology and Poison Control Centre Munich, Department of Internal Medicine II, School of Medicine, Technical University of Munich, Munich, Germany

\section{Tobias Zellner}

Division of Clinical Toxicology and Poison Control Centre Munich, Department of Internal Medicine II, School of Medicine, Technical University of Munich, Munich, Germany

\section{Armin Ott}

Technical University of Munich, Munich

\section{Florian Eyer}

Division of Clinical Toxicology and Poison Control Centre Munich, Department of Internal Medicine II, School of Medicine, Technical University of Munich, Munich, Germany

\section{Research}

Keywords: Suicide, self-poisoning, epidemiology, prevention, high-risk medication

Posted Date: May 11th, 2021

DOI: https://doi.org/10.21203/rs.3.rs-474643/v1

License: @ (i) This work is licensed under a Creative Commons Attribution 4.0 International License. Read Full License

Version of Record: A version of this preprint was published at International Journal of Mental Health Systems on January 25 th, 2022. See the published version at https://doi.org/10.1186/s13033-021-00513-8. 


\section{Abstract}

\section{Background}

Although the total number of suicides decreased since the beginning of the $80 \mathrm{~s}$, the number of suicide-related behaviors using selfintoxication increased. Therefore, research on the characteristics of individuals committing self-intoxication becomes of growing importance for risk assessments and the development of preventive measures.

\section{Methods}

In this prospective, observational, monocentric cohort study, all incoming calls at our Poisons Control Centre reporting suicide-related behavior through self-intoxication, were analyzed via a standardized questionnaire over 12 months. Both univariate and bivariate analyses were performed.

\section{Results}

1238 cases of deliberate intoxication were included in the study. The majority of cases occurred in the age group between 18 and 44 $(n=607 / 49 \%)$, two-thirds were female $(n=817 / 66 \%)$. The main substances used were antidepressants $(n=420 / 34 \%)$, peripheral analgesics $(n=322 / 26 \%)$ and neuroleptics $(n=282 / 23 \%)$. The majority of patients ingested substances from their prescribed medication ( $n=640 / 82 \%)$ with the highest proportion in the eldest $(n=72 / 113 ; 91 \%, p<0.001)$. Addiction was reported for the minority of patients ( $n=175 / 23 \%)$. For 704 cases $(57 \%)$, a psychiatric diagnosis was documented. Factors associated with recurrent suiciderelated behaviors were: i) an underlying psychiatric diagnosis ( $\mathrm{OR}=6.2$; $95 \% \mathrm{Cl} 3.8-10.4)$; ii) addiction problems $(\mathrm{OR}=2.4$; $95 \% \mathrm{Cl} 1.5$ $3.8)$; iii) ingestion of neuroleptics $(\mathrm{OR}=2.1,95 \%-\mathrm{Cl} 1.4-3.0)$ or antidepressants (OR=1.6; $95 \% \mathrm{Cl} 1.2-2.3)$.

\section{Conclusion}

This study might contribute to identifying individuals with an increased risk of suicide-related behaviors and to developing preventive strategies for future suicide attempt(s).

\section{Background}

The total number of suicides decreased in Germany since the beginning of the 80 s (1). Still, suicide is a more frequent cause of death than accidents, drug abuse, AIDS, and murder combined (2). After hanging, self-intoxication is the second most common method for suicide with increasing numbers of cases (3).

This emphasizes the importance of collecting reliable epidemiological data about characteristics of self-intoxication in the context of suicide-related behaviors (SRB), which - according to the definition of Silverman et al. 2007 - includes the terms self-harm, selfinflicted unintentional death, undetermined suicide-related behaviors, self-inflicted death with undetermined intent, suicide attempt and suicide (4). Identifying these characteristics may help to identify potential candidates for SRB and to derive preventive strategies, thereby supporting in forestalling preventable deaths. Current information on the characteristics of individuals showing SRB through self-intoxication is limited. Available studies focus either on information on substances used (5), substances used according to age group (6) or on individuals explicitly admitted to emergency care $(7,8,9)$. In another study the underlying psychiatric diseases are focused on (10). Further studies investigate more closely on the characteristics of individuals committing repeated suicide attempts (11). Accordingly, studies at hand focus on specific types of patients or specific parameters while our study presents information on unselected patients, thus offering information applicable to a broader range of subjects. The only study including parameter that are similar to the ones collected in the study at hand presents information collected between 1985 and 1997 (12).

In this study, the incoming calls at our Poisons Control Centre (PCC) were screened to prospectively collect data from a non-selected population in Germany. These data present a unique profile of SRB via self-intoxication within the German population, offering realworld evidence on characteristics of self-intoxicated suicides and suicide attempts.

\section{Methods}

\section{Design \& Setting}


The study was set up as a prospective, observational monocentric cohort study. All incoming calls to our PCC ( $1^{\text {st }}$ March 2017 to $28^{\text {th }}$ February 2018) reporting SRB through self-intoxication, independent of the outcome (fatal/non-fatal), were included (figure 1). These calls were initiated by emergency physicians, EMS personnel, emergency and critical care physicians, and occasionally by laypersons who were entrusted with the emergency care of persons with (para-)suicidal intoxications. The PCC is one of Germany's larger institutions, serving a population of approximately 13 million, and is contacted by lay people (50\%) as well as by clinicians (30\%) and general practitioners (15\%) seeking advice on poisonings of any kind. The data were collected by trained poison specialists, thus ensuring data quality. The study protocol was approved by the institutional review board (IRB) of the University Hospital (589/16 S). All methods were carried out in accordance with relevant guidelines and regulations.

\section{Data collection and inclusion criteria}

The calls were documented on a standardized protocol including type and amount of the ingested substance, time of ingestion, age, and gender of the patient as well as symptoms. Furthermore, the existence of an accompanying psychiatric diagnosis (PD) and previous SRB was enquired. If a PD was present, it was assigned to the $10^{\text {th }}$ revision of the International Statistical Classification of Diseases and Related Health Problems (ICD-10).

For the purpose of this study, questions about accessing the toxins, co-ingestion of ethanol or drugs, addiction disorders, history of suicide attempts and alleged reasons for the suicide attempt were added to the PCC's standard protocol (see supplement 1). At least two of these items as well as two items of the baseline characteristics (age, gender and ingested substance) were required for inclusion of the case. Due to the collection of pseudonymized data only, the requirement for patient informed consent was waived. Since the data were collected in a real world setting and were often only incomplete due to the emergency situation, many cases were excluded in advance because of the fragmentary information available.

Nonetheless, the callers were informed about the study and its content before being asked about the parameters of interest.

\section{Statistics}

Categorical variables are presented by absolute and relative frequencies referring to evaluable cases in each analysis. Group comparisons were performed by $\chi^{2}$-tests. If the number of expected cell counts was smaller than 5 , Fisher's exact test was used.

The level of statistical significance was assumed to be $p \leq 0.05$. Because of the exploratory character of this study, we did not adjust for multiple testing. Statistical analysis was performed using IBM SPSS Statistics for Windows, version 25 (IBM Corp., Armonk, N.Y., USA) and R version 3.5.2 (R Foundation for Statistical Computing, Vienna, Austria).

Multiple answers were possible regarding the ingested substances as well as the sources of supply. In this study, we generated binary variables (yes/no) for each substance as well as for each source of supply and ICD-10 classification for PD. Ranging from 12-95 years, the observed variation in age is considerable. Therefore, four age groups have been defined for evaluation purposes ( $<18$ years, 18-44 years, 45-64 years and $>64$ years).

\section{Results}

3720 cases showing SRB were recorded during the survey period. Each case represents one instance of SRB. 2482 cases were excluded due to incomplete data so that 1238 cases were included in the analysis (see figure 1). For the different sub-analyzes, the exact number of cases may vary due to missing data. For each analysis presented below, $100 \%$ refers to the totality of cases for which the respective parameter were available.

\section{Gender-specific outcomes}

The majority of cases were female $(n=817 ; 66 \%)$. The proportions of the age groups differed across gender $(p<0.001)$. The majority of cases in both gender groups were between $18-44$ years old. However, almost $13 \%(n=98)$ of the female population were $<18$ years old, whereas in the male population only $5 \%(n=18)$ were $<18$ years old. 
704 cases $(79 \%)$ had a PD, and affective disorders were the dominant PD (figure 2a). However, a gender-specific difference with regard to the frequency of a documented PD ( $n=491 ; 81 \%$ of all female cases vs. $n=211 ; 74 \%$ of all male cases, $p=0.03$ ) was detected. Men $(n=84,33 \%)$ were significantly more often affected by an addiction problem than women $(n=90,18 \%, p=<0.001)$. Alcohol was the most frequently used addictive substance in both groups $(n=115 ; 15.2 \%$; see table 1$)$.

Table 1 Clinical characteristics of suicide attempters with respect to gender distribution. 


\begin{tabular}{|c|c|c|c|c|c|c|}
\hline & & \multicolumn{5}{|l|}{ Gender } \\
\hline & & Male & Female & Total & Value & $\begin{array}{l}\text { Missing } \\
\text { data }\end{array}$ \\
\hline & & $\mathrm{n}(\%)$ & $\mathrm{n}(\%)$ & $\mathrm{n}(\%)$ & & Number \\
\hline \multirow[t]{5}{*}{ Age groups } & $<18$ & $18(4.6)$ & $98(12.9)$ & $116(10.1)$ & \multirow[t]{5}{*}{$<0.001$} & \multirow[t]{5}{*}{85} \\
\hline & $18-44$ & $216(55.2)$ & 391 (51.3) & $607(52.6)$ & & \\
\hline & $45-64$ & $112(28.6)$ & $206(27.0)$ & $318(27.6)$ & & \\
\hline & $>64$ & $45(11.6)$ & $67(8.8)$ & $112(9.7)$ & & \\
\hline & Total & $\begin{array}{l}391 \\
(100.0)\end{array}$ & $\begin{array}{l}762 \\
(100.0)\end{array}$ & $\begin{array}{l}1153 \\
(100.0)\end{array}$ & & \\
\hline \multirow[t]{3}{*}{ Psychiatric disorder } & Yes & $211(74.3)$ & $491(80.9)$ & $702(78.8)$ & \multirow[t]{3}{*}{0.031} & \multirow[t]{3}{*}{347} \\
\hline & No & $73(25.7)$ & $116(19.1)$ & $189(21.2)$ & & \\
\hline & Total & $\begin{array}{l}284 \\
(100.0)\end{array}$ & $\begin{array}{l}607 \\
(100.0)\end{array}$ & $891(100.0)$ & & \\
\hline \multirow[t]{6}{*}{ Addiction } & Alcohol & $54(21.2)$ & $61(12.1)$ & $115(15.2)$ & \multirow[t]{6}{*}{$<0.001$} & \multirow[t]{6}{*}{480} \\
\hline & Illicit drugs & $20(7.8)$ & $13(2.6)$ & $33(4.4)$ & & \\
\hline & $\begin{array}{l}\text { Multiple addictive } \\
\text { substances }\end{array}$ & $2(0.8)$ & $3(0.6)$ & $5(0.7)$ & & \\
\hline & Pharma-ceuticals & $8(3.1)$ & $13(2.6)$ & $21(2.8)$ & & \\
\hline & None & $171(67.1)$ & $413(82.1)$ & $584(77.0)$ & & \\
\hline & Total & $\begin{array}{l}255 \\
(100.0)\end{array}$ & $\begin{array}{l}503 \\
(100.0)\end{array}$ & $758(100.0)$ & & \\
\hline \multirow[t]{3}{*}{ History of suicide attempt } & Yes & $98(43.9)$ & $226(45.8)$ & $324(45.2)$ & \multirow[t]{3}{*}{0.659} & \multirow[t]{3}{*}{521} \\
\hline & No & $126(56.3)$ & $267(54.2)$ & $393(54.8)$ & & \\
\hline & Total & $\begin{array}{l}224 \\
(100.0)\end{array}$ & $\begin{array}{l}493 \\
(100.0)\end{array}$ & $717(100.0)$ & & \\
\hline \multirow{6}{*}{$\begin{array}{l}\text { Reasons for the suicide } \\
\text { attempt }\end{array}$} & Emotional act & $52(51.0)$ & $142(57.0)$ & $194(55.3)$ & \multirow[t]{6}{*}{0.156} & \multirow[t]{6}{*}{887} \\
\hline & Health problems & $11(10.8)$ & $15(6.0)$ & $26(7.4)$ & & \\
\hline & Conflicts at work & $7(6.9)$ & $6(2.4)$ & $13(3.7)$ & & \\
\hline & $\begin{array}{l}\text { Conflicts with family/ } \\
\text { partner }\end{array}$ & $23(22.5)$ & $63(25.3)$ & $86(24.5)$ & & \\
\hline & Others & $9(8.8)$ & 23 (92.) & $32(9.1)$ & & \\
\hline & Total & $\begin{array}{l}102 \\
(100.0)\end{array}$ & $\begin{array}{l}249 \\
(100.0)\end{array}$ & $351(100.0)$ & & \\
\hline \multicolumn{7}{|l|}{ Substances } \\
\hline \multirow[t]{3}{*}{ Peripheral analgesics } & Yes & $120(29.0)$ & $201(24.8)$ & $321(26.2)$ & \multirow[t]{3}{*}{0.137} & \multirow[t]{3}{*}{15} \\
\hline & No & $294(71.0)$ & $608(75.2)$ & $902(73.8)$ & & \\
\hline & Total & $\begin{array}{l}414 \\
(100.0)\end{array}$ & $\begin{array}{l}809 \\
(100.0)\end{array}$ & $\begin{array}{l}1223 \\
(100.0)\end{array}$ & & \\
\hline \multirow[t]{3}{*}{ Antidepressants } & Yes & $131(31.6)$ & $289(35.7)$ & $420(34.3)$ & \multirow[t]{3}{*}{0.174} & \multirow[t]{3}{*}{15} \\
\hline & No & $283(68.4)$ & $520(64.3)$ & $803(65.7)$ & & \\
\hline & & ge $5 / 17$ & & & & \\
\hline
\end{tabular}




\begin{tabular}{|c|c|c|c|c|c|c|}
\hline & Total & $\begin{array}{l}414 \\
(100.0)\end{array}$ & $\begin{array}{l}809 \\
(100.0)\end{array}$ & $\begin{array}{l}1223 \\
(100.0)\end{array}$ & & \\
\hline \multirow[t]{3}{*}{ Neuroleptics } & Yes & 80 (19.3) & $202(25.0)$ & $282(23.1)$ & \multirow[t]{3}{*}{0.032} & \multirow[t]{3}{*}{15} \\
\hline & No & 334 (80.7) & $607(75.0)$ & $941(76.9)$ & & \\
\hline & Total & $\begin{array}{l}414 \\
(100.0)\end{array}$ & $\begin{array}{l}809 \\
(100.0)\end{array}$ & $\begin{array}{l}1223 \\
(100.0)\end{array}$ & & \\
\hline \multirow{3}{*}{$\begin{array}{l}\text { Benzodiazepines } \\
\text { Z-drug }\end{array}$} & Yes & 85 (20.5) & $163(20.1)$ & $248(20.3)$ & \multirow[t]{3}{*}{0.934} & \multirow[t]{3}{*}{15} \\
\hline & No & $329(79.5)$ & 646 (79.9) & 975 (79.7) & & \\
\hline & Total & $\begin{array}{l}414 \\
(100.0)\end{array}$ & $\begin{array}{l}809 \\
(100.0)\end{array}$ & $\begin{array}{l}1223 \\
(100.0)\end{array}$ & & \\
\hline \multirow[t]{3}{*}{ Others } & Yes & 148 (35.7) & 256 (31.6) & $404(33.0)$ & \multirow[t]{3}{*}{0.149} & \multirow[t]{3}{*}{15} \\
\hline & No & $266(64.3)$ & $553(68.4)$ & $819(67.0)$ & & \\
\hline & Total & $\begin{array}{l}414 \\
(100.0)\end{array}$ & $\begin{array}{l}809 \\
(100.0)\end{array}$ & $\begin{array}{l}1223 \\
(100.0)\end{array}$ & & \\
\hline \multirow[t]{4}{*}{ Co-ingestion } & Alcohol & $\begin{array}{l}137 \\
(40.9)\end{array}$ & $186(28.6)$ & $323(32.8)$ & \multirow[t]{4}{*}{$<0.001$} & \multirow[t]{4}{*}{15} \\
\hline & Illicit drugs & $6(1.8)$ & $4(0.6)$ & $10(1.0)$ & & \\
\hline & None & $192(57.3)$ & $460(70.8)$ & $652(66.2)$ & & \\
\hline & Total & $\begin{array}{l}335 \\
(100.0)\end{array}$ & $\begin{array}{l}650 \\
(100.0)\end{array}$ & $985(100.0)$ & & \\
\hline \multirow[t]{3}{*}{ Own medication } & Yes & 218 (85.2) & $419(80.0)$ & 637 (81.7) & \multirow[t]{3}{*}{0.097} & \multirow[t]{3}{*}{458} \\
\hline & No & $38(14.8)$ & $105(20.0)$ & $143(18.3)$ & & \\
\hline & Total & $\begin{array}{l}256 \\
(100.0)\end{array}$ & $\begin{array}{l}524 \\
(100.0)\end{array}$ & $780(100.0)$ & & \\
\hline
\end{tabular}

\section{Age group-specific outcomes}

The age group-specific differences of the three most commonly ingested substance groups (peripheral analgesics, neuroleptics, benzodiazepines/Z-drugs) (figure 2b) were statistically significant (see table 3). For the age group <18 years, the usage of peripheral analgesics was highest $(n=40 ; 35 \%)$, while the $>64$-year-olds used benzodiazepines/Z-drugs most frequently $(n=37 ; 33 \%)$. The most common source of supply in all age groups was the patient's own medication (figure 2c), yet the older the age the higher the proportion $(p<0.001)$. A PD was documented less frequently in the $>64$-year-old group $(n=48 ; 59 \%, p<0.001)$.

Furthermore, the distribution of addictive problems varied with age $(\mathrm{p}<0.001)$. The $45-64$-year-olds showed the highest prevalence of addiction with around one third $(n=65 ; 33 \%)$. Except for those $<18$ years, the most frequently documented addictive substance in total was ethanol.

Age group-specific differences $(p<0.001)$ were found regarding the reasons for SRB. Except for the oldest group, where health problems were reported most frequently, an emotional act was dominant in all other age groups (figure $2 \mathrm{~d}$, see also table 2 and for the pairwise comparisons supplement 2).

Table 2 Clinical characteristics of suicide attempters with respect to age group distribution. 


\begin{tabular}{|c|c|c|c|c|c|c|c|c|}
\hline & & \multicolumn{7}{|c|}{ Age Groups } \\
\hline & & $<18$ & $18-44$ & $45-64$ & $>64$ & Total & $\begin{array}{l}\text { p- } \\
\text { Value }\end{array}$ & $\begin{array}{l}\text { Missing } \\
\text { data }\end{array}$ \\
\hline & & $\mathrm{n}(\%)$ & $\mathrm{n}(\%)$ & $\mathrm{n}(\%)$ & $\mathrm{n}(\%)$ & $\mathrm{n}(\%)$ & & \\
\hline \multirow[t]{3}{*}{ Psychiatric disorder } & Yes & $\begin{array}{l}71 \\
(78.9)\end{array}$ & 367 (80.7) & $\begin{array}{l}189 \\
(81.8)\end{array}$ & $\begin{array}{l}48 \\
(59.3)\end{array}$ & $\begin{array}{l}675 \\
(78.8)\end{array}$ & \multirow[t]{3}{*}{$<0.001$} & \multirow[t]{3}{*}{381} \\
\hline & No & $\begin{array}{l}19 \\
(21.1)\end{array}$ & 88 (19.3) & $\begin{array}{l}42 \\
(18.2)\end{array}$ & $\begin{array}{l}33 \\
(40.7)\end{array}$ & $\begin{array}{l}182 \\
(21.2)\end{array}$ & & \\
\hline & Total & $\begin{array}{l}90 \\
(100.0)\end{array}$ & $\begin{array}{l}455 \\
(100.0)\end{array}$ & $\begin{array}{l}231 \\
(100.0)\end{array}$ & $\begin{array}{l}81 \\
(100.0)\end{array}$ & $\begin{array}{l}857 \\
(100.0)\end{array}$ & & \\
\hline \multirow[t]{6}{*}{ Addiction } & Alcohol & $1(1.1)$ & $53(14.4)$ & $\begin{array}{l}52 \\
(26.7)\end{array}$ & $8(11.3)$ & $\begin{array}{l}114 \\
(15.8)\end{array}$ & \multirow[t]{6}{*}{$<0.001$} & \multirow[t]{6}{*}{515} \\
\hline & Illicit drugs & $2(2.3)$ & $25(6.8)$ & $4(2.1)$ & $0(0.0)$ & $31(4.3)$ & & \\
\hline & $\begin{array}{l}\text { Multiple addictive } \\
\text { substances }\end{array}$ & $0(0.0)$ & $4(1.1)$ & $1(0.5)$ & $0(0.0)$ & $5(0.7)$ & & \\
\hline & Medicines & $0(0.0)$ & $11(3.0)$ & $8(4.1)$ & $2(2.8)$ & $21(2.9)$ & & \\
\hline & None & $\begin{array}{l}85 \\
(96.6)\end{array}$ & $276(74.8)$ & $\begin{array}{l}130 \\
(66.7)\end{array}$ & $\begin{array}{l}61 \\
(85.9)\end{array}$ & $\begin{array}{l}552 \\
(76.3)\end{array}$ & & \\
\hline & Total & $\begin{array}{l}88 \\
(100.0)\end{array}$ & $\begin{array}{l}369 \\
(100.0)\end{array}$ & $\begin{array}{l}195 \\
(100.0)\end{array}$ & $\begin{array}{l}71 \\
(100.0)\end{array}$ & $\begin{array}{l}723 \\
(100.0)\end{array}$ & & \\
\hline \multirow[t]{3}{*}{$\begin{array}{l}\text { History of suicide } \\
\text { attempt }\end{array}$} & Yes & $\begin{array}{l}34 \\
(40.0)\end{array}$ & $182(51.3)$ & $\begin{array}{l}76 \\
(45.0)\end{array}$ & $\begin{array}{l}23 \\
(31.9)\end{array}$ & $\begin{array}{l}315 \\
(46.3)\end{array}$ & \multirow[t]{3}{*}{0.012} & \multirow[t]{3}{*}{557} \\
\hline & No & $\begin{array}{l}51 \\
(60.0)\end{array}$ & $173(48.7)$ & $\begin{array}{l}93 \\
(55.0)\end{array}$ & $\begin{array}{l}49 \\
(68.1)\end{array}$ & $\begin{array}{l}366 \\
(53.7)\end{array}$ & & \\
\hline & Total & $\begin{array}{l}85 \\
(100.0)\end{array}$ & $\begin{array}{l}355 \\
(100.0)\end{array}$ & $\begin{array}{l}169 \\
(100.0)\end{array}$ & $\begin{array}{l}72 \\
(100.0)\end{array}$ & $\begin{array}{l}681 \\
(100.0)\end{array}$ & & \\
\hline \multirow[t]{6}{*}{ Reasons } & Emotional act & $\begin{array}{l}23 \\
(57.5)\end{array}$ & $97(56.1)$ & $\begin{array}{l}57 \\
(63.3)\end{array}$ & $9(27.3)$ & $\begin{array}{l}186 \\
(55.4)\end{array}$ & \multirow[t]{6}{*}{$<0.001$} & \multirow[t]{6}{*}{902} \\
\hline & Health problems & $0(0.0)$ & $9(5.2)$ & $5(5.6)$ & $\begin{array}{l}11 \\
(33.3)\end{array}$ & $25(7.4)$ & & \\
\hline & Conflict at work & $5(12.5)$ & $7(4.0)$ & $1(1.1)$ & $0(0.0)$ & $13(3.9)$ & & \\
\hline & $\begin{array}{l}\text { Conflict with family/ } \\
\text { partner }\end{array}$ & $\begin{array}{l}11 \\
(27.5)\end{array}$ & $45(26.0)$ & $\begin{array}{l}20 \\
(22.2)\end{array}$ & $5(15.2)$ & $81(24.1)$ & & \\
\hline & Others & $1(2.5)$ & $15(8.7)$ & $7(7.8)$ & $8(24.2)$ & $31(9.2)$ & & \\
\hline & Total & $\begin{array}{l}40 \\
(100.0)\end{array}$ & $173(100.0)$ & $\begin{array}{l}90 \\
(100.0)\end{array}$ & $\begin{array}{l}33 \\
(100.0)\end{array}$ & $\begin{array}{l}336 \\
(100.0)\end{array}$ & & \\
\hline \multicolumn{9}{|l|}{ Substances } \\
\hline \multirow[t]{3}{*}{$\begin{array}{l}\text { Peripheral } \\
\text { analgesics }\end{array}$} & Yes & $\begin{array}{l}40 \\
(34.5)\end{array}$ & $175(28.8)$ & $\begin{array}{l}67 \\
(21.1)\end{array}$ & $\begin{array}{l}26 \\
(23.2)\end{array}$ & $\begin{array}{l}308 \\
(26.7)\end{array}$ & \multirow[t]{3}{*}{0.013} & \multirow[t]{3}{*}{86} \\
\hline & No & $\begin{array}{l}76 \\
(65.5)\end{array}$ & $432(71.2)$ & $\begin{array}{l}250 \\
(78.9)\end{array}$ & $\begin{array}{l}86 \\
(76.8)\end{array}$ & $\begin{array}{l}844 \\
(73.3)\end{array}$ & & \\
\hline & Total & $\begin{array}{l}116 \\
(100.0)\end{array}$ & $\begin{array}{l}607 \\
(100.0)\end{array}$ & $\begin{array}{l}317 \\
(100.0)\end{array}$ & $\begin{array}{l}112 \\
(100.0)\end{array}$ & $\begin{array}{l}1152 \\
(100.0)\end{array}$ & & \\
\hline \multirow[t]{2}{*}{ Antidepressants } & Yes & $\begin{array}{l}37 \\
(31.9)\end{array}$ & $211(34.8)$ & $\begin{array}{l}118 \\
(37.2)\end{array}$ & $\begin{array}{l}32 \\
(28.6)\end{array}$ & $\begin{array}{l}398 \\
(34.5)\end{array}$ & \multirow[t]{2}{*}{0.37} & \multirow[t]{2}{*}{86} \\
\hline & No & $\begin{array}{l}79 \\
(68.1)\end{array}$ & $396(65.2)$ & $\begin{array}{l}199 \\
(62.8)\end{array}$ & $\begin{array}{l}80 \\
(71.4)\end{array}$ & $\begin{array}{l}754 \\
(65.5)\end{array}$ & & \\
\hline
\end{tabular}




\begin{tabular}{|c|c|c|c|c|c|c|c|c|}
\hline & Total & $\begin{array}{l}116 \\
(100.0)\end{array}$ & $\begin{array}{l}607 \\
(100.0)\end{array}$ & $\begin{array}{l}317 \\
(100.0)\end{array}$ & $\begin{array}{l}112 \\
(100.0)\end{array}$ & $\begin{array}{l}1152 \\
(100.0)\end{array}$ & & \\
\hline \multirow[t]{3}{*}{ Neuroleptics } & Yes & $\begin{array}{l}29 \\
(25.0)\end{array}$ & $154(25.4)$ & $\begin{array}{l}72 \\
(22.7)\end{array}$ & $\begin{array}{l}15 \\
(13.4)\end{array}$ & $\begin{array}{l}270 \\
(23.4)\end{array}$ & \multirow[t]{3}{*}{0.05} & \multirow[t]{3}{*}{86} \\
\hline & No & $\begin{array}{l}87 \\
(75.0)\end{array}$ & 453 (74.6) & $\begin{array}{l}245 \\
(77.3)\end{array}$ & $\begin{array}{l}97 \\
(86.6)\end{array}$ & $\begin{array}{l}882 \\
(76.6)\end{array}$ & & \\
\hline & Total & $\begin{array}{l}116 \\
(100.0)\end{array}$ & $\begin{array}{l}607 \\
(100.0)\end{array}$ & $\begin{array}{l}317 \\
(100.0)\end{array}$ & $\begin{array}{l}112 \\
(100.0)\end{array}$ & $\begin{array}{l}1152 \\
(100.0)\end{array}$ & & \\
\hline \multirow{3}{*}{$\begin{array}{l}\text { Benzodiazepines/ } \\
\text { Z-drugs }\end{array}$} & Yes & $5(4.3)$ & $114(18.8)$ & $\begin{array}{l}76 \\
(24.0)\end{array}$ & $\begin{array}{l}37 \\
(33.0)\end{array}$ & $\begin{array}{l}232 \\
(20.1)\end{array}$ & \multirow[t]{3}{*}{$<0.001$} & \multirow[t]{3}{*}{86} \\
\hline & No & $\begin{array}{l}111 \\
(95.7)\end{array}$ & $493(81.2)$ & $\begin{array}{l}241 \\
(76.0)\end{array}$ & $\begin{array}{l}75 \\
(67.0)\end{array}$ & $\begin{array}{l}920 \\
(79.9)\end{array}$ & & \\
\hline & Total & $\begin{array}{l}116 \\
(100.0)\end{array}$ & $\begin{array}{l}607 \\
(100.0)\end{array}$ & $\begin{array}{l}317 \\
(100.0)\end{array}$ & $\begin{array}{l}112 \\
(100.0)\end{array}$ & $\begin{array}{l}1152 \\
(100.0)\end{array}$ & & \\
\hline \multirow[t]{3}{*}{ Others } & Yes & $\begin{array}{l}39 \\
(33.6)\end{array}$ & $187(30.8)$ & $\begin{array}{l}108 \\
(34.1)\end{array}$ & $\begin{array}{l}47 \\
(42.0)\end{array}$ & $\begin{array}{l}381 \\
(33.1)\end{array}$ & \multirow[t]{3}{*}{0.135} & \multirow[t]{3}{*}{86} \\
\hline & No & $\begin{array}{l}77 \\
(66.4)\end{array}$ & $420(69.2)$ & $\begin{array}{l}209 \\
(65.9)\end{array}$ & $\begin{array}{l}65 \\
(58.0)\end{array}$ & $\begin{array}{l}771 \\
(66.9)\end{array}$ & & \\
\hline & Total & $\begin{array}{l}116 \\
(100.0)\end{array}$ & $\begin{array}{l}607 \\
(100.0)\end{array}$ & $\begin{array}{l}317 \\
(100.0)\end{array}$ & $\begin{array}{l}112 \\
(100.0)\end{array}$ & $\begin{array}{l}1152 \\
(100.0)\end{array}$ & & \\
\hline \multirow[t]{4}{*}{ Co-ingestion } & Alcohol & $5(5.2)$ & $186(37.8)$ & $\begin{array}{l}101 \\
(40.6)\end{array}$ & $\begin{array}{l}14 \\
(15.1)\end{array}$ & $\begin{array}{l}306 \\
(32.9)\end{array}$ & \multirow[t]{4}{*}{$<0.001$} & \multirow[t]{4}{*}{86} \\
\hline & Illicit drugs & $1(1.0)$ & $8(1.6)$ & $1(0.4)$ & $0(0.0)$ & $10(1.1)$ & & \\
\hline & None & $\begin{array}{l}90 \\
(93.8)\end{array}$ & $298(60.6)$ & $\begin{array}{l}147 \\
(59.0)\end{array}$ & $\begin{array}{l}79 \\
(84.9)\end{array}$ & $\begin{array}{l}614 \\
(66.0)\end{array}$ & & \\
\hline & Total & $\begin{array}{l}96 \\
(100.0)\end{array}$ & $\begin{array}{l}492 \\
(100.0)\end{array}$ & $\begin{array}{l}249 \\
(100.0)\end{array}$ & $\begin{array}{l}93 \\
(100.0)\end{array}$ & $\begin{array}{l}930 \\
(100.0)\end{array}$ & & \\
\hline \multirow[t]{3}{*}{ Own medication } & Yes & $\begin{array}{l}50 \\
(60.2)\end{array}$ & 297 (80.7) & $\begin{array}{l}181 \\
(87.0)\end{array}$ & $\begin{array}{l}72 \\
(91.1)\end{array}$ & $\begin{array}{l}600 \\
(81.3)\end{array}$ & \multirow[t]{3}{*}{$<0.001$} & \multirow[t]{3}{*}{500} \\
\hline & No & $\begin{array}{l}33 \\
(39.8)\end{array}$ & 71 (19.3) & $\begin{array}{l}27 \\
(13.0)\end{array}$ & $7(8.9)$ & $\begin{array}{l}138 \\
(18.7)\end{array}$ & & \\
\hline & Total & $\begin{array}{l}83 \\
(100.0)\end{array}$ & $\begin{array}{l}368 \\
(100.0)\end{array}$ & $\begin{array}{l}208 \\
(100.0)\end{array}$ & $\begin{array}{l}79 \\
(100.0)\end{array}$ & $\begin{array}{l}738 \\
(100.0)\end{array}$ & & \\
\hline
\end{tabular}

\section{Repeated instances of Suicide-Related Behavior}

In 325 out of 718 (45\%) cases with complete data, at least one prior instance of SRB was recorded. The frequency of repeated instances of SRB varied with age (see table 2). Moreover, observations with prior instances of SRB suffered more often from a PD $(p<0.001)$ and from addiction problems than those without prior instances of SRB $(p<0.001)$. They also showed an increased use of neuroleptics $(p<0.001)$ and antidepressants $(p=0.003)$ and a less frequent use of peripheral analgesics $(p=0.034)($ see table 3$)$.

Table 3 History of Suicide attempts via self-intoxication. Assessment of different implications on repeated suicide. 


\begin{tabular}{|c|c|c|c|c|c|c|}
\hline & & \multicolumn{5}{|c|}{ History of suicide attempts } \\
\hline & & Yes & No & Total & p- & Missing data \\
\hline & & $\mathrm{n}(\%)$ & $\mathrm{n}(\%)$ & $\mathrm{n}(\%)$ & & \\
\hline \multirow[t]{3}{*}{ Psychiatric disorder } & Yes & $257(91.1)$ & $201(62.2)$ & $458(75.7)$ & \multirow[t]{3}{*}{$<0.001$} & \multirow[t]{3}{*}{633} \\
\hline & No & $25(8.9)$ & $122(37.8)$ & $147(24.3)$ & & \\
\hline & Total & $\begin{array}{l}282 \\
(100.0)\end{array}$ & $\begin{array}{l}323 \\
(100.0)\end{array}$ & $605(100.0)$ & & \\
\hline \multirow[t]{6}{*}{ Addiction } & Alcohol & $45(18.3)$ & $23(7.5)$ & $68(12.3)$ & \multirow[t]{6}{*}{$<0.001$} & \multirow[t]{6}{*}{684} \\
\hline & Illicit drugs & $11(4.5)$ & $9(2.9)$ & $20(3.6)$ & & \\
\hline & $\begin{array}{l}\text { Multiple addictive } \\
\text { substances }\end{array}$ & $1(0.4)$ & $2(0.6)$ & $3(0.5)$ & & \\
\hline & Medicines & $8(3.3)$ & $5(1.6)$ & $13(2.3)$ & & \\
\hline & None & $181(73.6)$ & $269(87.3)$ & $450(81.2)$ & & \\
\hline & Total & $\begin{array}{l}246 \\
(100.0)\end{array}$ & $\begin{array}{l}308 \\
(100.0)\end{array}$ & $\begin{array}{l}554 \\
(100.0)\end{array}$ & & \\
\hline \multirow[t]{6}{*}{ Reasons } & Affective action & $56(58.9)$ & $87(55.1)$ & $143(56.5)$ & \multirow[t]{6}{*}{0.366} & \multirow[t]{6}{*}{985} \\
\hline & Health problems & $8(8.4)$ & $12(7.6)$ & $20(7.9)$ & & \\
\hline & Conflict at work & $4(4.2)$ & $7(4.4)$ & $11(4.3)$ & & \\
\hline & Conflict with family/partner & $17(17.9)$ & $43(27.2)$ & $60(23.7)$ & & \\
\hline & Others & $10(10.5)$ & $9(5.7)$ & $19(7.5)$ & & \\
\hline & Total & $95(100.0)$ & $\begin{array}{l}158 \\
(100.0)\end{array}$ & $\begin{array}{l}253 \\
(100.0)\end{array}$ & & \\
\hline \multicolumn{7}{|l|}{ Substances } \\
\hline \multirow[t]{3}{*}{ Peripheral analgesics } & Yes & $77(23.8)$ & $122(31.2)$ & $199(27.8)$ & \multirow[t]{3}{*}{0.034} & \multirow[t]{3}{*}{523} \\
\hline & No & $247(76.2)$ & $269(68.8)$ & $516(72.2)$ & & \\
\hline & Total & $\begin{array}{l}324 \\
(100.0)\end{array}$ & $\begin{array}{l}391 \\
(100.0)\end{array}$ & $\begin{array}{l}715 \\
(100.0)\end{array}$ & & \\
\hline \multirow[t]{3}{*}{ Antidepressants } & Yes & $120(37.0)$ & $103(26.3)$ & $223(31.2)$ & \multirow[t]{3}{*}{0.003} & \multirow[t]{3}{*}{523} \\
\hline & No & $204(63.0)$ & $288(73.7)$ & $492(68.8)$ & & \\
\hline & Total & $\begin{array}{l}324 \\
(100.0)\end{array}$ & $\begin{array}{l}391 \\
(100.0)\end{array}$ & $\begin{array}{l}715 \\
(100.0)\end{array}$ & & \\
\hline \multirow[t]{3}{*}{ Neuroleptics } & Yes & $91(28.1)$ & $62(15.9)$ & $153(21.4)$ & \multirow[t]{3}{*}{$<0.001$} & \multirow[t]{3}{*}{523} \\
\hline & No & 233 (71.9) & $329(84.1)$ & $562(78.6)$ & & \\
\hline & Total & $\begin{array}{l}324 \\
(100.0)\end{array}$ & $\begin{array}{l}391 \\
(100.0)\end{array}$ & $\begin{array}{l}715 \\
(100.0)\end{array}$ & & \\
\hline \multirow{3}{*}{$\begin{array}{l}\text { Benzodiazepines/Z- } \\
\text { Drugs }\end{array}$} & Yes & $66(20.4)$ & $82(21.0)$ & $148(20.7)$ & \multirow[t]{3}{*}{0.916} & \multirow[t]{3}{*}{523} \\
\hline & No & $258(79.6)$ & $309(79.0)$ & $567(79.3)$ & & \\
\hline & Total & $\begin{array}{l}324 \\
(100.0)\end{array}$ & $\begin{array}{l}391 \\
(100.0)\end{array}$ & $\begin{array}{l}715 \\
(100.0)\end{array}$ & & \\
\hline \multirow[t]{2}{*}{ Others } & Yes & $121(37.3)$ & $119(30.4)$ & $240(33.6)$ & \multirow[t]{2}{*}{0.051} & \multirow[t]{2}{*}{523} \\
\hline & No & $203(62.7)$ & $272(69.6)$ & $475(66.4)$ & & \\
\hline
\end{tabular}




\begin{tabular}{|c|c|c|c|c|c|c|}
\hline & Total & $\begin{array}{l}324 \\
(100.0)\end{array}$ & $\begin{array}{l}391 \\
(100.0)\end{array}$ & $\begin{array}{l}715 \\
(100.0)\end{array}$ & & \\
\hline \multirow[t]{4}{*}{ Co-ingestion } & Alcohol & $93(33.3)$ & $94(29.1)$ & 187 (31.1) & \multirow[t]{4}{*}{0.544} & \multirow[t]{4}{*}{636} \\
\hline & Illicit drugs & $2(0.7)$ & $3(0.9)$ & $5(0.8)$ & & \\
\hline & None & 184 (65.9) & $226(70.0)$ & $410(68.1)$ & & \\
\hline & Total & $\begin{array}{l}279 \\
(100.0)\end{array}$ & $\begin{array}{l}323 \\
(100.0)\end{array}$ & $\begin{array}{l}602 \\
(100.0)\end{array}$ & & \\
\hline \multirow[t]{3}{*}{ Own medication } & Yes & 185 (86.4) & 199 (73.7) & 384 (79.3) & \multirow[t]{3}{*}{0.001} & \multirow[t]{3}{*}{754} \\
\hline & No & $29(13.6)$ & $71(26.3)$ & $100(20.7)$ & & \\
\hline & Total & $\begin{array}{l}214 \\
(100.0)\end{array}$ & $\begin{array}{l}270 \\
(100.0)\end{array}$ & $\begin{array}{l}484 \\
(100.0)\end{array}$ & & \\
\hline
\end{tabular}

\section{Discussion}

The main findings of this study can be summarized as follows: 1) The majority of instances of SRB occurred in the age-group between 18 and 44. 2) Two-thirds of the reported cases were women. 3) Antidepressants, peripheral analgesics and neuroleptics were the main substances used for self-intoxication. 4) Most often, the substances came from the patient's own medication. 5) Although addiction played a relevant role, the majority of cases had no reported addiction disease. The most commonly abused substance was ethanol. 6) Factors associated with recurrent instances of SRB were: i) an underlying PD; ii) addiction problems; iii) the ingestion of antidepressants or neuroleptics.

Two thirds of the analyzed cases were female, and the proportion was particularly high among the < 18-year olds, which is in line with data from other studies $(10,12,16,20,21,29,33)$. Spiller et al. showed that females constituted nearly $71 \%$ of all cases of selfintoxication in patients younger than 25 years (6). The high proportion of females may surprise, as the majority of completed suicides is performed by males (13-15). However, both our study and the study conducted by Spiller et al. focus on self-intoxication, which is the preferred method for females when displaying $\operatorname{SRB}(18,19)$. Furthermore, the present study shows, that most cases of selfintoxication are performed in the age group between 18 and 44 years, which is in accordance with further studies on this subject (17).

Antidepressants constitute the most commonly used substance group followed by peripheral analgesics. The latter were predominantly used by younger individuals, which is also reflected in the existing literature $(6,25,26)$. In contrast to many other studies, in which benzodiazepines are usually ranked among the three most popular substances $(7,17,22,28-30,33)$, they ranged only on fourth place in our investigation. A similar pattern could be detected regarding paracetamol, which ranged as the most common single substance in international studies (28, 31 and also Table 4), but was only the fourth common in our study. Instead, ibuprofen was the most common single substance, which is important as ibuprofen has a high mortality rate (in large overdoses) despite its perceived harmlessness $(27,32)$. As the study at hand presents a profile of the characteristics of individuals displaying SRB through self-intoxication in Germany, the relatively uncommon use of benzodiazepines and paracetamol might reflect a more geographic preference or could indicate a change in prescription behavior.

Almost $80 \%$ of the cases had a prevalence of PD, which is higher compared to data found in literature $(18,29,33)$. This might be partly biased by the method of data acquisition, as the diagnosis of a PD was mostly not recorded by a psychiatrist.

Females seemed to suffer more often from an accompanying PD. This finding is in line with data from Ghazinour et al. (29). In contrast, Prescott et al. found no gender-specific difference and Mauri et al. found gender-specific differences concerning specific PDs only $(18,33)$.

Focusing more closely on the types of PD, depression dominated by far. This corresponds to findings from other studies showing that $65-90 \%$ of SRB relating to a PD involve depression (19). Concerning the cases with repeated instances of SRB, a similar pattern could be identified $(\mathrm{OR}=6.2 ; 95 \% \mathrm{Cl} 3.8-10.4)$. Among those, depression was also frequent $(\mathrm{OR}=1.4 ; 95 \% \mathrm{Cl} 1.0-1.9)$.

This aspect together with the increased usage of antidepressants and neuroleptics might explain the relatively high percentage of cases with a history instances of SRB exceeding the findings in literature $(29,33)$. A PD and the usage of antidepressants and 
neuroleptics seem associated with repeated suicide attempts (17).

The strong association of addictive disorders and (recurrent) instances of SRB demonstrated in the literature $(23,24)$ is also supported by the present study in which approximately one-third of the cases of cases with repeated suicide-attempts had an addiction history $(\mathrm{OR}=2.5 ; 95 \% \mathrm{Cl} 1.6-4.0)$.

Furthermore, in line with existing data, the proportion of ethanol addiction is particularly high for cases with recurrent instances of SRB $(\mathrm{OR}=2.8 ; 95 \% \mathrm{Cl} 1.6-5.0)(22)$.

Alongside the finding that the presence of a PD is associated with an increased risk of further SRB, the presence of an (ethanol) addiction seems to constitute a second risk factor for recurrent SRB. Additionally, this study showed that the use of neuroleptics and antidepressants is frequent for cases with repeated instances of SRB. In combination with the finding by Pfeifer et al. who showed that antidepressants, if tricyclic, have a high mortality rate (5), the results could serve to identify potential risk patients at an early stage and to initiate appropriate preventive strategies such as closer monitoring according to their needs. This could involve e.g. psychological intervention (11) or aid to abstinence to reduce impulsivity, a better follow-up observance $(23,24)$ or a responsible prescription behavior. 
Table 4

Overview about literature research with respect to self-intoxication in correlation to age, gender, and substances used

\begin{tabular}{|c|c|c|c|c|c|c|c|}
\hline Autor/Year & Study design & Country & Sample size & $\begin{array}{l}\text { Median } \\
\text { age in } \\
\text { years } \\
\text { (range) }\end{array}$ & Male & Female & $\begin{array}{l}\text { Main substances } \\
\text { used }\end{array}$ \\
\hline $\begin{array}{l}\text { Hendrix L et } \\
\text { al., } 2012 \\
(29)\end{array}$ & $\begin{array}{l}\text { Retrospective } \\
\text { study }\end{array}$ & Belgium & 312 & 37 & $\begin{array}{l}122 \\
(39.1 \%)\end{array}$ & $\begin{array}{l}190 \\
(60.9 \%)\end{array}$ & $\begin{array}{l}\text { Benzodiazepine } \\
(51 \%), \\
\text { antidepressants } \\
(23 \%) \text {, antipsychotics } \\
(16 \%)\end{array}$ \\
\hline $\begin{array}{l}\text { Prescott K } \\
\text { et al., } 2009 \\
\text { (18) }\end{array}$ & $\begin{array}{l}\text { Retrospective } \\
\text { study }\end{array}$ & UK & 2665 & $\begin{array}{l}32(16- \\
90)\end{array}$ & $40.8 \%$ & $59.2 \%$ & $\begin{array}{l}\text { Paracetamol (43\%), } \\
\text { ibuprofen (17\%). }\end{array}$ \\
\hline $\begin{array}{l}\text { Bilén } \mathrm{K} \text { et } \\
\text { al., } 2011 \\
(17)\end{array}$ & $\begin{array}{l}\text { Retrospective } \\
\text { study }\end{array}$ & Sweden & 1524 & 39.5 & $35 \%$ & $65 \%$ & $\begin{array}{l}\text { Benzodiazepine } \\
(45 \%), \\
\text { antidepressants } \\
(16 \%), \text { paracetamol } \\
(16 \%)\end{array}$ \\
\hline $\begin{array}{l}\text { Kordrostami } \\
\text { et al., } 2017 \\
\text { (34) }\end{array}$ & $\begin{array}{l}\text { Forensic } \\
\text { toxicology } \\
\text { analysis }\end{array}$ & Iran & $\begin{array}{l}674 \\
\text { (completed } \\
\text { suicides) }\end{array}$ & 32.61 & $\begin{array}{l}462 \\
(68.55 \%)\end{array}$ & $\begin{array}{l}212 \\
(31.45 \%)\end{array}$ & $\begin{array}{l}\text { Aluminium } \\
\text { phosphide tablets } \\
\text { (pesticides), opioids, } \\
\text { methamphetamine }\end{array}$ \\
\hline $\begin{array}{l}\text { Salles et al., } \\
2018(22)\end{array}$ & $\begin{array}{l}\text { Retrospective } \\
\text { study }\end{array}$ & France & 516 & 42 & $\begin{array}{l}165 \\
(31.9 \%)\end{array}$ & $\begin{array}{l}351 \\
(68.1 \%)\end{array}$ & $\begin{array}{l}\text { Benzodiazepine } \\
(85 \%), \text { paracetamol } \\
(9 \%), \text { opioids }(4 \%)\end{array}$ \\
\hline $\begin{array}{l}\text { Marahatta } \\
\text { et al., } 2009 \\
\text { (33) }\end{array}$ & $\begin{array}{l}\text { Prospective } \\
\text { study }\end{array}$ & Nepal & 54 & $\begin{array}{l}29.87 \\
\text { (females), } \\
35.54 \\
\text { (males) }\end{array}$ & $42.6 \%$ & $57,4 \%$ & $\begin{array}{l}\text { Organophosphorus } \\
(74 \%), \\
\text { antidepressants } \\
(17 \%)\end{array}$ \\
\hline $\begin{array}{l}\text { Mauri et al., } \\
2005(10)\end{array}$ & $\begin{array}{l}\text { Prospective } \\
\text { study }\end{array}$ & Italy & 201 & 40 & $73(36 \%)$ & $128(64 \%)$ & $\begin{array}{l}\text { Benzodiazepine } \\
(59 \%), \text { neuroleptics } \\
(13 \%), \\
\text { antidepressants } \\
(13 \%)\end{array}$ \\
\hline $\begin{array}{l}\text { Cook et al., } \\
2008 \text { (28) }\end{array}$ & $\begin{array}{l}\text { Prospective } \\
\text { study }\end{array}$ & $\begin{array}{l}\text { UK, } \\
\text { Scotland }\end{array}$ & 530 & 33 & $35 \%$ & $65 \%$ & $\begin{array}{l}\text { Paracetamol (39\%), } \\
\text { antidepressants } \\
(35 \%), \\
\text { hypnotics/anxiolytics } \\
(25 \%)\end{array}$ \\
\hline $\begin{array}{l}\text { Ghazinour } \\
\text { et al., } 2009 \\
\text { (9) }\end{array}$ & $\begin{array}{l}\text { Prospective } \\
\text { study }\end{array}$ & Iran & $\begin{array}{l}2025 \\
\text { (parasuicides) }\end{array}$ & $\begin{array}{l}25.4 \\
\text { (females), } \\
28.5 \\
\text { (males) }\end{array}$ & $\begin{array}{l}966 \\
(47.7 \%)\end{array}$ & $\begin{array}{l}1059 \\
(52.3 \%)\end{array}$ & $\begin{array}{l}\text { Psychotropic drugs } \\
\text { and anticonvulsants } \\
(56 \%)\end{array}$ \\
\hline $\begin{array}{l}\text { Sorge et al., } \\
2015 \text { (16) }\end{array}$ & $\begin{array}{l}\text { Prospective } \\
\text { study }\end{array}$ & Germany & 3533 & $\begin{array}{l}35 \\
\text { (males), } \\
29 \\
\text { (females) }\end{array}$ & $62.6 \%$ & $37.4 \%$ & $\begin{array}{l}\text { Benzodiazepine } \\
(29 \%), \\
\text { antidepressants } \\
(20 \%) \text {, } \\
\text { antihistamines (13\%) }\end{array}$ \\
\hline \multirow[t]{2}{*}{$\begin{array}{l}\text { Michel et } \\
\text { al., } 1994 \\
(30)\end{array}$} & \multirow[t]{2}{*}{$\begin{array}{l}\text { Prospective } \\
\text { study }\end{array}$} & \multirow[t]{2}{*}{ Switzerland } & $\begin{array}{l}\text { Completed } \\
\text { suicide: } 179\end{array}$ & $\begin{array}{l}\text { Completed } \\
\text { suicide: } 53\end{array}$ & $\begin{array}{l}\text { Completed } \\
\text { suicide: } \\
49 \%\end{array}$ & $\begin{array}{l}\text { Completed } \\
\text { suicide: } \\
51 \%\end{array}$ & \multirow{2}{*}{$\begin{array}{l}\text { Benzodiazepine (CS: } \\
44 \% \text {, AS: } 46 \% \text { ), } \\
\text { antidepressants (CS: } \\
16 \% \text {, AS: } 11 \% \text { ) }\end{array}$} \\
\hline & & & $\begin{array}{l}\text { Attempted } \\
\text { suicide: } 269\end{array}$ & $\begin{array}{l}\text { Attempted } \\
\text { suicide: } 36\end{array}$ & $\begin{array}{l}\text { Attempted } \\
\text { suicide: } \\
35 \%\end{array}$ & $\begin{array}{l}\text { Attempted } \\
\text { suicide: } \\
65 \%\end{array}$ & \\
\hline
\end{tabular}

\section{Study Limitations}

Albeit the relatively large cohort, this study has several important limitations: It displays data from incoming calls in the real-life situation of acute PCC advisory. This means that information might be incomplete and no follow-up on the outcome of the individual 
showing SRB is available. Thereby, no discrimination between non-fatal and fatal outcome is possible.

Furthermore, classification of psychiatric diagnoses was not made by a specialist in psychiatry but on the basis of anamnestic information provided by the patient or his relatives, or on the basis of existing medical records.

Moreover, for some variables, e.g. the reason for displaying SRB, data are available for only approximately half of the cases. Interpretation of the data has therefore to be carried out cautiously.

\section{Conclusion}

The study delineates several characteristics of individuals displaying SRB by means of self-intoxication based on data collected from an unselected population in the real-world scenario of a PCC. It identifies differences based on gender, age, and recurrence of instances of SRB factors that are not only of considerable importance for the development of preventive measures but also allow for individual adaptation and use of the measures. The analysis shows that different characteristics are associated with an increased risk of SRB particularly the risk for recurrent instances of SRB. Hence, our findings may serve as a basis for future research, both on a national and international level to verify the characteristics identified here and offer further guidance for preventive strategies of suicides.

\section{Abbreviations}

IRB: Institutional Review Board

PCC: Poison Control Centre

PD: Psychiatric Disease

SRB: Suicide-Related Behavior

\section{Declarations}

\section{Acknowledgements}

Not applicable

\section{Funding}

This research did not receive any specific grant from funding agencies in the public, commercial, or not-for-profit sectors.

\section{Availability of data and materials}

All data generated or analyzed during this study are included in this published article. The datasets used and/or analyzed during the current study are available from the corresponding author on reasonable request.

\section{Ethics approval and consent to participate}

This study was approved by the IRB of the University Hospital (589/16 S). Due to the collection of anonymized data an informed consent to participate was formally waived by the IRB of the University Hospital.

\section{Competing interests}

The authors declare that they have no competing interests. 


\section{Consent for publication}

Not applicable

\section{Authors' contributions}

SG initiated the study, performed data entry, and wrote the manuscript. CD and AO performed the statistical analysis of the data. CR contributed with analysis and interpretation of data and revising the article of important intellectual content. TZ and FE made substantial contributions to the interpretation of data and manuscript review. All authors read and approved the final manuscript.

\section{Author' information}

${ }^{1}$ Division of Clinical Toxicology and Poison Control Centre Munich, Department of Internal Medicine II, School of Medicine, Technical University of Munich, Munich, Germany; Stefanie.Geith@tum.de.

${ }^{2}$ Department of Sociology, University of Munich (LMU), 80801 Munich; Christiane.Didden@soziologie.uni-muenchen.de

${ }^{3}$ Division of Clinical Toxicology and Poison Control Centre Munich, Department of Internal Medicine II, School of Medicine, Technical University of Munich, Munich, Germany; Christian.Rabe@tum.de

${ }^{4}$ Division of Clinical Toxicology and Poison Control Centre Munich, Department of Internal Medicine II, School of Medicine, Technical University of Munich, Munich, Germany; Tobias.Zellner@tum.de

${ }^{5}$ Staburo GmbH, Aschauer Str. 26b, 81549 Munich, Germany; ott@staburo.de

${ }^{6}$ Division of Clinical Toxicology and Poison Control Centre Munich, Department of Internal Medicine II, School of Medicine, Technical University of Munich, Munich, Germany; Florian.Eyer@tum.de

\section{References}

1. Statista.com. Durchschnittliche Anzahl von Suiziden in Deutschland in ausgewählten Jahren von 1920 bis 2014 (je eine Millionen Einwohner) https://de.statista.com/statistik/daten/studie/596120/umfrage/durchschnittliche-anzahl-von-suiziden-indeutschland/2016. Accessed on 31 July 2020

2. statista.com. Anzahl der Suizide in Deutschland im Vergleich zu ausgewählten Todesursachen in den Jahren 2012 bis 2014. Accessed on 31 July 2020 https://de.statista.com/statistik/daten/studie/318378/umfrage/anzahl-der-suizide-in-deutschland-imvergleich-zu-ausgewaehlten-todesursachen/2016. Accessed on 31 July 2020

3. Fiedler G. Gesundheitsberichterstattung des Bundes. Statistisches Bundesamt, www.gbe-bund.de, Datenblätter vom 06.12.2012. Accessed on 31 July 2020

4. Silverman MM, Berman AL, Sanddal ND, O'Carroll PW, Joiner TE. Rebuilding the Tower of Babel: a revised nomenclature for the study of suicide and suicidal behaviors-Part 2: suicide-related ideations, communications, and behaviors. Suicide LifeThreat Behav. 2007;37:264-77

5. Pfeifer P, Greusing S, Kupferschmidt H, Bartsch C, Reisch T. A comprehensive analysis of attempted and fatal suicide cases involving frequently used psychotropic medications. Gen Hosp Psychiatry 2019

6. Spiller HA, Ackerman JP, Smith GA, Kistamgari S, Funk AR, McDermott MR, et al. Suicide attempts by self-poisoning in the United States among 10-25 year olds from 2000 to 2018: substances used, temporal changes and demographics. Clin Toxicol (Phila) 2019:1-12.

7. Sorge M, Weidhase L, Bernhard M, Gries A, Petros S. Self-poisoning in the acute care medicine 2005-2012. Anaesthesist 2015;64(6):456-62

8. Hendrix L, Verelst S, Desruelles D, Gillet JB. Deliberate self-poisoning: characteristics of patients and impact on the emergency department of a large university hospital. Emerg Med J. 2013;30(1):e9 
9. Marahatta SB, Singh J, Shrestha R, Koju R. Poisoning cases attending emergency department in Dhulikhel Hospital-Kathmandu University Teaching Hospital. Kathmandu Univ Med J (KUMJ). 2009;7(26):152-6

10. Haw C, Hawton K, Houston K, Townsend E. Psychiatric and personality disorders in deliberate self-harm patients. Br J Psychiatry 2001;178(1):48-54

11. Oh SH, Park KN, Jeong SH, Kim HJ, Lee CC. Deliberate self-poisoning: factors associated with recurrent self-poisoning. Am J Emerg Med 2011;29(8):908-12

12. Townsend E, Hawton K, Harriss L, Bale E, Bond A. Substances used in deliberate self-poisoning 1985-1997: trends and associations with age, gender, repetition and suicide intent. Soc Psychiatry Psychiatr Epidemio/ 2001;36(5):228-34

13. Robert Koch-Institut H. GBE-Schwerpunktbericht: Gesundheit von Frauen und Männern im mittleren Lebensalter. Gesundheitsberichterstattung des Bundes RKI, Berlin 2006

14. Mergl R, Koburger N, Heinrichs K, Szekely A, Toth MD, Coyne J, et al. What Are Reasons for the Large Gender Differences in the Lethality of Suicidal Acts? An Epidemiological Analysis in Four European Countries. PloS one 2015;10(7):e0129062

15. Kim B, Ahn JH, Cha B, Chung YC, Ha TH, Hong Jeong S, et al. Characteristics of methods of suicide attempts in Korea: Korea National Suicide Survey (KNSS). J Affect Disord 2015;188:218-25

16. Ajdacic-Gross V, Weiss MG, Ring M, Hepp U, Bopp M, Gutzwiller F, et al. Methods of suicide: international suicide patterns derived from the WHO mortality database. Bull World Health Organ 200;86(9):726-32

17. Bilen K, Ottosson C, Castren M, Ponzer S, Ursing C, Ranta P, et al. Deliberate self-harm patients in the emergency department: factors associated with repeated self-harm among 1524 patients. Emerg Med J 2011;28(12):1019-25

18. Prescott K, Stratton R, Freyer A, Hall I, Le Jeune I. Detailed analyses of self-poisoning episodes presenting to a large regional teaching hospital in the UK. Br J Clin Pharmacol 2009;68(2):260-8

19. Krug EG, Dahlberg LL, Mercy JA, Zwi AB, Lozano R. World report on violence and health. World Health Organization. 2002

20. Schmidtke A, Bille-Brahe U, DeLeo D, Kerkhof A, Bjerke T, Crepet P, et al. Attempted suicide in Europe: rates, trends and sociodemographic characteristics of suicide attempters during the period 1989-1992. Results of the WHO/EURO Multicentre Study on Parasuicide. Acta Psychiatr Scand 1996;93(5):327-38

21. Hawton K, Fagg J, Simkin S, Bale E, Bond A. Deliberate self-harm in adolescents in Oxford, 1985-1995. J Adolesc 2000;23(1):4755

22. Salles J, Calonge J, Franchitto N, Bougon E, Schmitt L. Factors associated with hospitalization after self-poisoning in France: special focus on the impact of alcohol use disorder. BMC Psychiatry. 2018;18(1):287

23. Ruusa J, Bergman B, Sundell ML. Alcohol Withdrawal, Psychological Symptoms, and Treatment Success. Subst Abus 2000;21(3):129-35

24. Davidson CL, Wingate LR, Rasmussen KA, Slish ML. Hope as a predictor of interpersonal suicide risk. Suicide Life Threat Behav. 2009;39(5):499-507

25. Kim J, Kim M, Kim YR, Choi KH, Lee KU. High Prevalence of Psychotropics Overdose among Suicide Attempters in Korea. Clinical psychopharmacology and neuroscience: the official scientific journal of the Korean College of Neuropsychopharmacology. 2015;13(3):302-7

26. Geith S, Ganzert M, Schmoll S, Acquarone D, Deters M, Sauer O, et al. [Intoxications in Children and Adolescents in Germany]. Klinische Padiatrie. 2018;230(4):205-14

27. Geith S, Renner B, Rabe C, Stenzel J, Eyer F. Ibuprofen plasma concentration profile in deliberate ibuprofen overdose with circulatory depression treated with therapeutic plasma exchange: a case report. BMC Pharmacol Toxicol 2017;18(1):81

28. Cook R, Allcock R, Johnston M. Self-poisoning: current trends and practice in a U.K. teaching hospital. Clin Med (Lond) 2008;8(1):37-40

29. Ghazinour M, Emami H, Richter J, Abdollahi M, Pazhumand A. Age and gender differences in the use of various poisoning methods for deliberate parasuicide cases admitted to loghman hospital in Tehran (2000-2004). Suicide Life Threat Behav 2009;39(2):231-9

30. Michel K, Arestegui G, Spuhler T. Suicide with psychotropic drugs in Switzerland. Pharmacopsychiatry 1994;27(3):114-8

31. Neeleman J, Wessely S. Drugs taken in fatal and non-fatal self-poisoning: a study in south London. Acta Psychiatr Scand $1997 ; 95(4): 283-7$

Page $15 / 17$ 
32. Kato H, Yoshimoto K, Ikegaya H. Two cases of oral aspirin overdose. J Forensic Leg Med 2010;17(5):280-2

33. Mauri MC, Cerveri G, Volonteri LS, Fiorentini A, Colasanti A, Manfre S, et al. Parasuicide and drug self-poisoning: analysis of the epidemiological and clinical variables of the patients admitted to the Poisoning Treatment Centre (CAV), Niguarda General Hospital, Milan. Clin Pract Epidemiol Ment Health 2005;1(1):5

34. Kordrostami R, Akhgari M, Ameri M, Ghadipasha M, Aghakhani K. Forensic toxicology analysis of self-poisoning suicidal deaths in Tehran, Iran; trends between 2011-2015. Daru 2017;25(1):15

Figures

\section{PCC}

(serving a population of approximately 13.000 .000 people):

37.700 incoming calls between

\subsubsection{7 to 28.02 .2018}


\section{Figure 1}

Data flow 


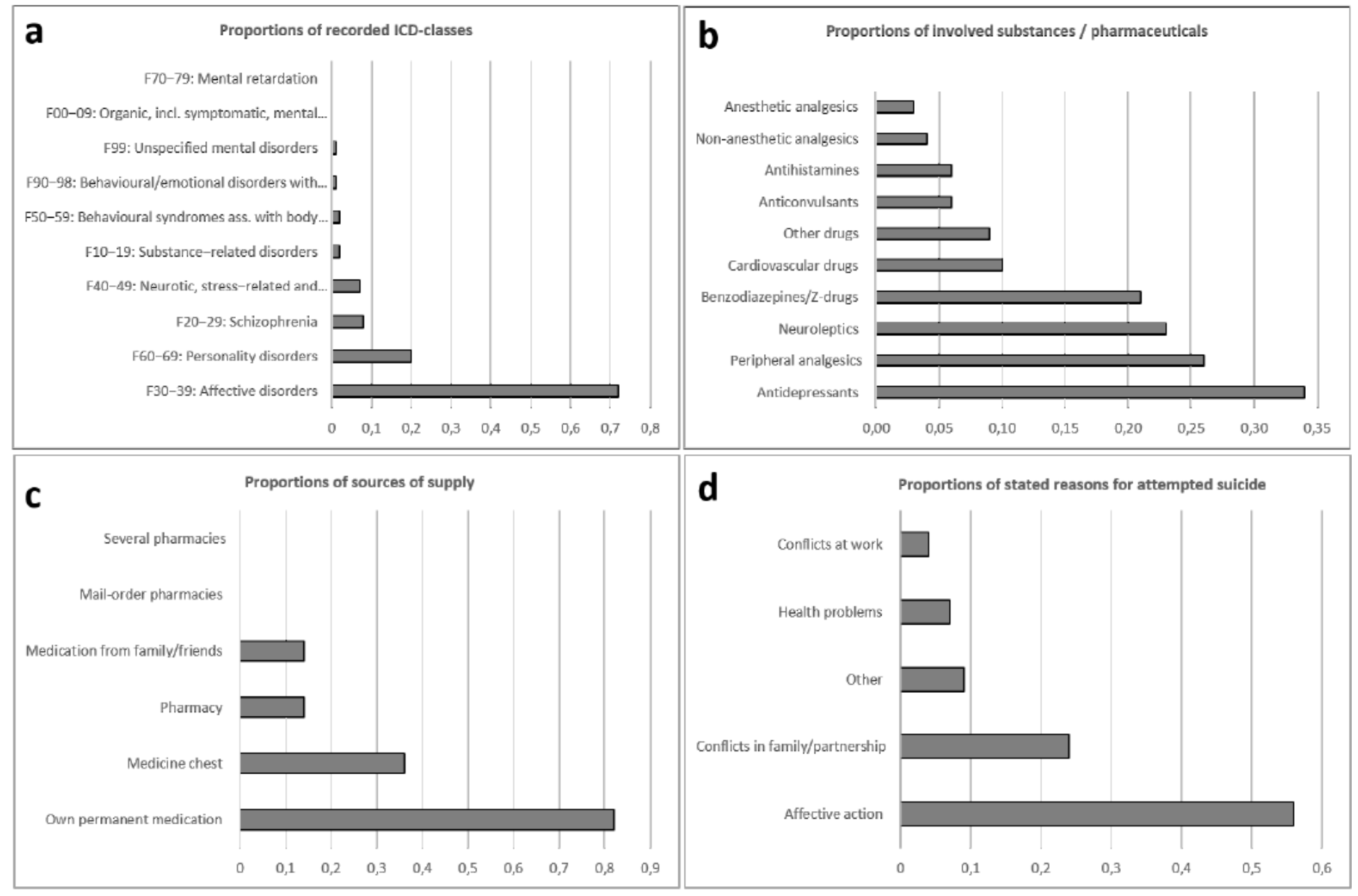

\section{Figure 2}

Overview of psychiatric disorders, frequently used substances, sources of supply, and reasons for suicide. a) Psychiatric disorders according to the ICD10-classification, b) Most frequently used substances, c) Sources of supply and d) Most frequent reasons for suicide (attempt). Multiple answers were possible.

\section{Supplementary Files}

This is a list of supplementary files associated with this preprint. Click to download.

- Geithetal2021Supplement1.pdf

- Geithetal2021Supplement2.pdf 